ESAIM: M2AN 48 (2014) 1413-1429

DOI: $10.1051 / \mathrm{m} 2 \mathrm{an} / 2014001$
ESAIM: Mathematical Modelling and Numerical Analysis

www.esaim-m2an.org

\title{
ERROR ESTIMATES FOR STOKES PROBLEM WITH TRESCA FRICTION CONDITIONS
}

\author{
Mekki Ayadi ${ }^{1}$, Leonardo Baffico ${ }^{2}$, Mohamed Khaled Gdoura ${ }^{1,2}$ \\ AND TAOUFIK SASSI ${ }^{2}$
}

\begin{abstract}
In this paper, we present and study a mixed variational method in order to approximate, with the finite element method, a Stokes problem with Tresca friction boundary conditions. These non-linear boundary conditions arise in the modeling of mold filling process by polymer melt, which can slip on a solid wall. The mixed formulation is based on a dualization of the non-differentiable term which define the slip conditions. Existence and uniqueness of both continuous and discrete solutions of these problems is guaranteed by means of continuous and discrete inf-sup conditions that are proved. Velocity and pressure are approximated by $P 1$ bubble-P1 finite element and piecewise linear elements are used to discretize the Lagrange multiplier associated to the shear stress on the friction boundary. Optimal a priori error estimates are derived using classical tools of finite element analysis and two uncoupled discrete inf-sup conditions for the pressure and the Lagrange multiplier associated to the fluid shear stress.
\end{abstract}

Mathematics Subject Classification. 45N30, 76D07, 35J87, 35M87.

Received February 2, 2012. Revised November 18, 2013.

Published online August 13, 2014.

\section{INTRODUCTION}

No-slip hypothesis at fluid-wall interface leads to good agreement with experimental observations for Newtonian fluids which is no longer true for non-Newtonian fluid [27]. For example, in the flow of certain high molecular weight linear polymers through circular dies, the exit flow rate has been found to be a discontinuous function of pressure drop over a certain range of shear rates [22]. This observation is consistent with the hypothesis that the velocity at the wall does not vanish. Several studies have been made and showed not only that slip takes place when a threshold is reached but also it's the origin of many defects and instabilities in the polymer injection process [32]. The Tresca boundary conditions used in this paper could model this phenomenon, since it states, in one hand, that when the magnitude of the fluid shear stress, on some part of the fluid boundary, reaches a given threshold value, then the tangential fluid velocity may be different to zero, i.e. fluid may slip on this part of the boundary. In the other hand, when the fluid shear stress magnitude is strictly below the threshold

Keywords and phrases. Stokes problem, Tresca friction, variational inequality, mixed finite element, error estimates.

1 Université Tunis El Manar, Laboratoire de Modélisation Mathématiques et Numérique dans les Sciences de l'Ingénieur, Ecole Nationale d'Ingénieurs de Tunis, B.P. 32, 1002 Tunis, Tunisie.

mekki.ayadi@enis.rnu.tn; mohamedkhaled.gdoura@lamsin.rnu.tn

2 Université de Caen Basse-Normandie, Laboratoire de Mathématiques Nicolas Oresme, CNRS UMR 6139, UFR sciences

Campus II, Bd Maréchal JUIN, 14032 Caen Cedex, France. leonardo.baffico@unicaen.fr; taoufik.sassi@unicaen.fr 
value, then the tangential velocity is zero. These boundary conditions are completed with a non penetration condition (i.e. normal velocity is equal to zero). Let us mention that a slip boundary condition could be used also in modeling the blood flow through a diseased artery (see [31] and the reference therein).

The first attempt to integrate this kind of boundary conditions in a numerical simulation of a fluid flow is due to Doltsinis et al. [12] and Fortin and Côté [14]. Since that, many papers were published simulating various flows with such boundary conditions, see [29] and references therein. In [18], numerical results were obtained using the augmented Lagrangian method and a block relaxation algorithm.

The mathematical analysis of this class of non linear boundary conditions, that leads to variational inequality problems, has been introduced by Fujita in [15], where he investigated some hydrodynamics problems with leak and slip boundary conditions involving subdifferential property. In [16], Fujita studied the variational inequality of the second kind formulation (where the velocity and pressure are the only unknowns) of the Stokes problem with slip boundary conditions which leads to a non-differentiable problem. He established the existence and uniqueness of a weak solution to the resulting problem. Saito in $[30,31]$ studied the regularity of Fujita's weak solution. Recently, based on the penalty method, finite element approximation of the non-differentiable problem introduced by Fujita has been proposed in [25] and several error estimates with strong regularity assumption on the velocity field are obtained.

The aim of this work is to contribute to the numerical analysis of Stokes problems with Tresca (slip) boundary conditions in the two dimensional case. Our first purpose is to introduce another Lagrange multiplier related to the tangential component of the fluid stress on the slip zone, that will transform the non-differentiable problem into a smooth one, and to prove that this new formulation has a unique solution. This method has been previously used in the study of contact problems with friction in elasticity (see for example [23]). In the present work, we adapt it in order to take into account the presence of two Lagrange multipliers: the fluid pressure, associated to the incompressibility condition and defined in the whole domain, and the tangential component of the fluid stress, defined only on a part of the fluid domain boundary. Our second goal is to propose a mixed finite element approximation of the continuous three-field mixed problem and to carry out the convergence analysis in order to obtain optimal a priori error estimates.

The paper is organized as follows. In Section 2, the main notations and the functional framework are presented. In Section 3, we introduce the equations modeling the Stokes problem with Tresca boundary conditions. Then, in Section 4, we establish and study a continuous mixed variational formulation. In this section a coupled continuous inf-sup condition, involving the fluid pressure and the new Lagrange multiplier associated to the Tresca boundary conditions, is proved. Using this result an existence and uniqueness result is established in the continuous case. The following section is devoted to study a mixed finite element approximation and a coupled discrete inf-sup condition is proved, which allows us to state an existence and uniqueness result for the discrete formulation. In Section 6, a priori error estimates are derived. We show an optimal convergence order of $h^{3 / 4}$ with $\mathbf{H}^{2}(\Omega)$ regularity assumption on the velocity.

\section{Preliminary and notations}

We need to set some notations and recall some functional tools necessary for our analysis. Let $\Omega \subset \mathbb{R}^{2}$ be an open bounded set with Lipschitz boundary $\partial \Omega$.

In what follows, the Euclidean norm of a point $\mathbf{x} \in \mathbb{R}^{2}$ is denoted by $|\mathbf{x}|$. The Lebesgue space $L^{2}(\Omega)$ is endowed with the norm:

$$
\|p\|_{0}=\left(\int_{\Omega}|p(\mathbf{x})|^{2} \mathrm{~d} \Omega\right)^{\frac{1}{2}}, \quad \forall p \in L^{2}(\Omega),
$$

corresponding to the scalar product:

$$
(p, q)=\int_{\Omega} p(\mathbf{x}) q(\mathbf{x}) \mathrm{d} \Omega
$$


while $L_{0}^{2}(\Omega)$ is the closed subspace of $L^{2}(\Omega)$ defined by:

$$
L_{0}^{2}(\Omega)=\left\{p \in L^{2}(\Omega) \text { such that } \int_{\Omega} p(\mathbf{x}) \mathrm{d} \Omega=0\right\} .
$$

We denote by $L^{\infty}(\Omega)$ the space of bounded functions on $\Omega$ which will be endowed with the norm

$$
\|\psi\|_{L^{\infty}(\Omega)}=\operatorname{ess} \sup _{\mathbf{x} \in \Omega}|\psi(\mathbf{x})|, \quad \forall \psi \in L^{\infty}(\Omega) .
$$

We make constant use of the standard Sobolev space $H^{m}(\Omega), m \geq 1$, provided with the norm:

$$
\|\psi\|_{m}=\left(\sum_{0 \leq|\alpha| \leq m}\left\|\partial^{\alpha} \psi\right\|_{0}^{2}\right)^{1 / 2}
$$

where $\alpha$ is a multi-index.

Fractional Sobolev spaces $H^{\varrho}(\Omega), \varrho \in \mathbb{R}_{+} \backslash \mathbb{N}$ are defined by

$$
H^{\varrho}(\Omega)=\left\{\varphi \in H^{m}(\Omega) \text { such that }\|\varphi\|_{\varrho, \Omega}<+\infty\right\},
$$

with

$$
\|\varphi\|_{\varrho, \Omega}=\left(\|\varphi\|_{m}^{2}+\sum_{|\alpha|=m} \int_{\Omega} \int_{\Omega} \frac{\left(\partial^{\alpha} \varphi(\mathbf{x})-\partial^{\alpha} \varphi(\mathbf{y})\right)^{2}}{|\mathbf{x}-\mathbf{y}|^{2+2 \theta}} \mathrm{d} \Omega_{\mathbf{x}} \mathrm{d} \Omega_{\mathbf{y}}\right)^{\frac{1}{2}}
$$

with $m$ being the integer part of $\varrho$ and $\theta$ its decimal parts.

The closure in $H^{\varrho}(\Omega)$ of $\mathcal{D}(\Omega)$, the space of infinitely differentiable functions with compact support in $\Omega$, is denoted by $H_{0}^{\varrho}(\Omega)$.

On any measurable part $\Gamma \subseteq \partial \Omega$ we introduce the space $H^{\frac{1}{2}}(\Gamma)$ as follows

$$
H^{\frac{1}{2}}(\Gamma)=\left\{\varphi \in L^{2}(\Gamma) \text { such that }\|\varphi\|_{\frac{1}{2}, \Gamma}<+\infty\right\},
$$

where

$$
\|\psi\|_{\frac{1}{2}, \Gamma}=\left(\|\psi\|_{0, \Gamma}^{2}+\int_{\Gamma} \int_{\Gamma} \frac{(\psi(\mathbf{x})-\psi(\mathbf{y}))^{2}}{|\mathbf{x}-\mathbf{y}|^{2}} \mathrm{~d} \Gamma_{\mathbf{x}} \mathrm{d} \Gamma_{\mathbf{y}}\right)^{\frac{1}{2}} \quad \text { and } \quad\|\psi\|_{0, \Gamma}=\left(\int_{\Gamma}|\psi(s)|^{2} \mathrm{~d} \Gamma\right)^{\frac{1}{2}}
$$

We denote by $H_{00}^{\frac{1}{2}}(\Gamma)$ the subspace of $H^{\frac{1}{2}}(\Gamma)$ given in Lions and Magenes [26] or in Kikuchi and Oden [24] (see Chap. 5, p. 84). The topological dual space of $H^{\frac{1}{2}}(\Gamma)$ (respectively $H_{00}^{\frac{1}{2}}(\Gamma)$ ) is denoted $H^{-\frac{1}{2}}(\Gamma)$ (respectively $\left.H_{*}^{-\frac{1}{2}}(\Gamma)\right)$ and we denote by $\langle\cdot, \cdot\rangle$ the duality pairing. The norm in $H^{-\frac{1}{2}}(\Gamma)$ is defined by

$$
\|\mu\|_{-\frac{1}{2}, \Gamma}=\sup _{\varphi \in H^{\frac{1}{2}}(\Gamma), \varphi \neq 0} \frac{\langle\mu, \varphi\rangle}{\|\varphi\|_{\frac{1}{2}, \Gamma}}, \quad \forall \mu \in H^{-\frac{1}{2}}(\Gamma) .
$$

If there is no confusion we use the same notation for $H_{*}^{-\frac{1}{2}}(\Gamma)$ norm, i.e.

$$
\|\mu\|_{-\frac{1}{2}, \Gamma}=\sup _{\varphi \in H_{00}^{\frac{1}{2}}(\Gamma), \varphi \neq 0} \frac{\langle\mu, \varphi\rangle}{\|\varphi\|_{\frac{1}{2}, \Gamma}}, \quad \forall \mu \in H_{*}^{-\frac{1}{2}}(\Gamma) .
$$


The Cartesian product of $d$ previous spaces and their elements are denoted by bold character. The respective norms are introduced as follows:

$$
\begin{aligned}
& \|\mathbf{v}\|_{m}=\left(\sum_{i=1}^{d}\left\|v_{i}\right\|_{m}^{2}\right)^{1 / 2} \mathbf{v}=\left(v_{1}, \ldots, v_{d}\right) \in \mathbf{H}^{m}(\Omega), \\
& \|\mathbf{w}\|_{\frac{1}{2}, \Gamma}=\left(\sum_{i=1}^{d}\left\|w_{i}\right\|_{\frac{1}{2}, \Gamma}^{2}\right)^{1 / 2} \mathbf{w}=\left(w_{1}, \ldots, w_{d}\right) \in \mathbf{H}^{\frac{1}{2}}(\Gamma), \\
& \|\boldsymbol{\mu}\|_{-\frac{1}{2}, \Gamma}=\left(\sum_{i=1}^{d}\left\|\mu_{i}\right\|_{-\frac{1}{2}, \Gamma}^{2}\right)^{1 / 2} \quad \boldsymbol{\mu}=\left(\mu_{1}, \ldots, \mu_{d}\right) \in \mathbf{H}^{-\frac{1}{2}}(\Gamma) .
\end{aligned}
$$

Let $\mathcal{X} \subset H^{1}(\Omega)$ be a subspace of functions vanishing on an open non-empty portion $\Gamma_{0} \subset \partial \Omega, \operatorname{meas}\left(\Gamma_{0}\right)>0$ :

$$
\mathcal{X}=\left\{v \in H^{1}(\Omega) \text { such that } v_{\left.\right|_{\Gamma_{0}}}=0\right\} .
$$

Let $d=2$. We introduce the energetic norm $\||\cdot|\|_{1}$ in $\mathcal{X}$ corresponding to the scalar product

$$
(\mathbf{u}, \mathbf{v})_{1}=\int_{\Omega} \sum_{i, j=1}^{d} \varepsilon_{i j}(\mathbf{u}) \varepsilon_{i j}(\mathbf{v}) \mathrm{d} \Omega,
$$

where $\varepsilon_{i j}(\mathbf{u})$ is the $i j$ th component of the linearized strain rate tensor

$$
\varepsilon(\mathbf{u})=\frac{1}{2}\left(\nabla \mathbf{u}+\nabla^{t} \mathbf{u}\right) .
$$

From the Korn's inequality it follows that $\|\cdot\|_{1}$ and $\||\cdot|\|_{1}$ are equivalent in $\mathcal{X}$.

We denote by $\mathbf{n}$ (resp. t) the outward unit normal vector (resp. unit tangent vector) to $\partial \Omega$ and $u_{n}$, respectively $u_{t}$, the normal, respectively the tangential, component of $\mathbf{u}$.

The stress vector is equal to $\boldsymbol{\sigma} \mathbf{n}$ where $\boldsymbol{\sigma}$ is the Cauchy stress tensor defined by:

$$
\boldsymbol{\sigma}=2 \nu \varepsilon(\mathbf{u})-p \boldsymbol{\delta},
$$

where $p$ is the hydrostatic pressure, $\boldsymbol{\delta}$ is the identity tensor and $\nu$ is the kinematic fluid viscosity. We denote by $\sigma_{n}$ (resp. $\sigma_{t}$ ) the normal (resp. tangential) component of $\boldsymbol{\sigma n}$, i.e. $\sigma_{n}=\boldsymbol{\sigma} \mathbf{n} . \mathbf{n}$ and $\sigma_{t}=\boldsymbol{\sigma}$ n.t.

Remark 2.1. We use the notation $u_{t}$ and $\sigma_{t}$ for the tangential components of the velocity and the stress vector since in the two-dimensional case $(d=2)$, they are scalar quantities.

\section{Setting The STOKES PROBLEM WITH NONLINEAR BOUNDARY CONDITIONS}

Let us consider a convex open bounded set $\Omega \subset \mathbb{R}^{2}$. The boundary $\partial \Omega$ is the union of two non overlapping portions $\Gamma_{0}$ and $\Gamma$ such that $\bar{\Gamma} \cap \bar{\Gamma}_{0}=\varnothing$. No-slip boundary conditions are prescribed on $\Gamma_{0}$ while $\Gamma$ is where the fluid may slip. The formulation of the Stokes problem with nonlinear boundary conditions of Tresca friction type consists in finding a velocity vector field $\mathbf{u}$ and a pressure $p$ satisfying the following equations and conditions:

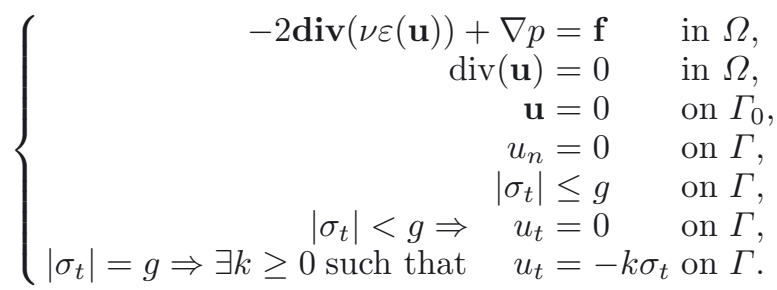


Here $\mathbf{f} \in \mathbf{L}^{2}(\Omega)$ is the external volume force acting on the fluid, and $g \in L^{\infty}(\Gamma), g \geq 0$ a.e., is the threshold function.

The next result is needed to derive the variational problem (see [5]).

Proposition 3.1. The following equivalence holds on $\Gamma$ :

$$
\left\{\begin{array} { c } 
{ | \sigma _ { t } | < g \Rightarrow u _ { t } = 0 } \\
{ | \sigma _ { t } | = g \Rightarrow \exists k \geq 0 \text { s.t. } u _ { t } = - k \sigma _ { t } }
\end{array} \Longleftrightarrow \quad \left\{\begin{array}{l}
\left|\sigma_{t}\right| \leq g \\
\sigma_{t} u_{t}+g\left|u_{t}\right|=0 .
\end{array}\right.\right.
$$

Then, using (3.2), one can derive the variational formulation of (3.1):

$$
\left\{\begin{array}{l}
\text { Find }(\mathbf{u}, p) \in \mathbf{V}_{\operatorname{div}}(\Omega) \times L_{0}^{2}(\Omega) \text { such that } \forall \mathbf{v} \in \mathbf{V}(\Omega) \\
a(\mathbf{u}, \mathbf{v}-\mathbf{u})-(p, \operatorname{div}(\mathbf{v}))+j(\mathbf{v})-j(\mathbf{u}) \geq L(\mathbf{v}-\mathbf{u})
\end{array}\right.
$$

with

$$
\begin{gathered}
\mathbf{V}(\Omega)=\left\{\mathbf{v} \in \mathbf{H}^{1}(\Omega), \mathbf{v}_{\mid \Gamma_{0}}=0, \mathbf{v} \cdot \mathbf{n}_{\mid \Gamma}=0\right\} \\
\mathbf{V}_{\operatorname{div}}(\Omega)=\{\mathbf{v} \in \mathbf{V}(\Omega), \operatorname{div}(\mathbf{v})=0 \text { in } \Omega\} \\
a(\mathbf{u}, \mathbf{v})=\int_{\Omega} 2 \nu \varepsilon(\mathbf{u}): \varepsilon(\mathbf{v}) \mathrm{d} \Omega \\
L(\mathbf{v})=\int_{\Omega} \mathbf{f} \cdot \mathbf{v} \mathrm{d} \Omega
\end{gathered}
$$

and

$$
j(\mathbf{v})=\int_{\Gamma} g\left|v_{t}\right| \mathrm{d} \Gamma
$$

The existence and uniqueness of a solution to (3.3) was established in [16] while a regularity result was proved in $[30,31]$ :

Theorem 3.2. Problem (3.3) has a solution $(\mathbf{u}, p) \in \mathbf{V}_{\mathrm{div}}(\Omega) \times L^{2}(\Omega)$. Moreover, $\mathbf{u}$ is unique.

In addition, if $\int_{\Omega} p \mathrm{~d} \Omega=0$, i.e. $p \in L_{0}^{2}(\Omega)$, then, it comes that $p$ is unique.

In addition, if $\Gamma_{0}$ and $\Gamma$ are connected components of class $\mathscr{C}^{2}$ and $\mathscr{C}^{3}$, respectively, and $g \in L^{\infty}(\Gamma) \cap H^{1}(\Gamma)$ then it comes that $(\mathbf{u}, p) \in \mathbf{H}^{2}(\Omega) \times H^{1}(\Omega)$ with

$$
\|\mathbf{u}\|_{2}+\|p\|_{1} \leq C\|\boldsymbol{f}\|_{0}+\|g\|_{1, \Gamma}
$$

Since the bilinear form $a(\cdot, \cdot)$ is symmetric the variational problem $(3.3)$ is equivalent to the following constrained non-differentiable minimization problem:

$$
\left\{\begin{array}{l}
\text { Find } \mathbf{u} \in \mathbf{V}_{\mathrm{div}}(\Omega) \text { such that } \\
\mathcal{J}(\mathbf{u}) \leq \mathcal{J}(\mathbf{v}) \quad \forall \mathbf{v} \in \mathbf{V}_{\operatorname{div}}(\Omega)
\end{array}\right.
$$

where $\mathcal{J}(\mathbf{v})=\frac{1}{2} a(\mathbf{v}, \mathbf{v})+j(\mathbf{v})-L(\mathbf{v})$. 


\section{Mixed FORMulation}

In one hand, in order to solve (3.4) a Lagrange multiplier $q$ is needed to enforce the condition $\operatorname{div}(\mathbf{u})=0$ in $\Omega$, which can be identified with the pressure. In the other hand, Fujita proved in [17] that (3.3) is equivalent to

$$
\left\{\begin{array}{l}
\text { Find } \sigma_{t} \in H^{-\frac{1}{2}}(\Gamma) \text { such that }\left|\sigma_{t}\right| \leq g \text { on } \Gamma \text { and } \\
\int_{\Gamma} \sigma_{t}\left(v_{t}-u_{t}\right) \mathrm{d} \Gamma+j(\mathbf{v})-j(\mathbf{u}) \geq 0 \quad \forall \mathbf{v} \in \mathbf{V}(\Omega) .
\end{array}\right.
$$

The unknown function $\sigma_{t}$ is seen as a Lagrange multiplier and can be identified with the shear stress on $\Gamma$. Then, the minimization problem (3.4) is equivalent to the following saddle-point formulation:

$$
\left\{\begin{array}{l}
\text { Find }(\mathbf{u},(p, \lambda)) \in \mathscr{H} \quad \text { such that: } \\
\mathscr{L}(\mathbf{u},(q, \mu)) \leq \mathscr{L}(\mathbf{u},(p, \lambda)) \leq \mathscr{L}(\mathbf{v},(p, \lambda)) \quad \forall(\mathbf{v},(q, \mu)) \in \mathscr{H}
\end{array}\right.
$$

where

$$
\begin{gathered}
\mathscr{L}(\mathbf{v},(q, \mu))=\frac{1}{2} a(\mathbf{v}, \mathbf{v})-\int_{\Omega} q \operatorname{div}(\mathbf{v}) \mathrm{d} \Omega+\int_{\Gamma} \mu v_{t} \mathrm{~d} \Gamma-L(\mathbf{v}), \\
\mathscr{H}=\mathbf{V}(\Omega) \times L_{0}^{2}(\Omega) \times \mathcal{Q}
\end{gathered}
$$

and

$$
\mathcal{Q}=\left\{\mu \in L^{2}(\Gamma),|\mu| \leq g \text { a.e. }\right\}
$$

endowed with the $H_{*}^{-\frac{1}{2}}(\Gamma)$-norm. According to [23], we have

$$
\mathcal{Q}=\left\{\mu \in L^{2}(\Gamma), \int_{\Gamma} \mu \psi \mathrm{d} \Gamma-\int_{\Gamma} g|\psi| \mathrm{d} \Gamma \leq 0 \quad \forall \psi \in L^{2}(\Gamma)\right\} .
$$

The solution of problem (4.2) is characterized by (see $[2,24]$ ):

$$
\left\{\begin{array}{r}
\text { Find }(\mathbf{u},(p, \lambda)) \in \mathbf{V}(\Omega) \times \Lambda \quad \text { such that } \\
\qquad \begin{array}{r}
a(\mathbf{u}, \mathbf{v})+b((p, \lambda), \mathbf{v})=L(\mathbf{v}) \forall \mathbf{v} \in \mathbf{V}(\Omega) \\
b((q-p, \mu-\lambda), \mathbf{u}) \leq 0 \quad \forall(q, \mu) \in \Lambda,
\end{array}
\end{array}\right.
$$

with

$$
b((p, \lambda), \mathbf{v})=-(p, \operatorname{div}(\mathbf{v}))+\int_{\Gamma} \lambda v_{t} \mathrm{~d} \Gamma,
$$

and $\Lambda=L_{0}^{2}(\Omega) \times \mathcal{Q}$, which is a closed convex of $\mathcal{M}=L_{0}^{2}(\Omega) \times L^{2}(\Gamma)$.

To ensure the existence and uniqueness of a solution to (4.3), the following inf-sup condition is needed (see for example [6]):

Lemma 4.1. If $\partial \Omega$ is regular enough, then there exists a constant $\alpha>0$ such that: $\forall(q, \mu) \in \mathcal{M}$

$$
\sup _{\mathbf{v} \in \mathbf{V}(\boldsymbol{\Omega})} \frac{b((q, \mu), \mathbf{v})}{\|\mathbf{v}\|_{1}} \geq \alpha\left(\|q\|_{0}+\|\mu\|_{-\frac{1}{2}, \Gamma}\right) .
$$

Proof. We note that, similarly as in the study of a Signorini's problem for incompressible materials (see [24]), in order to obtain (4.5) it would be enough to prove that for all $(q, \mu) \in \mathcal{M}$ there exists $\mathbf{u} \in \mathbf{V}(\Omega)$ such that:

$$
\left\{\begin{aligned}
\operatorname{div} \mathbf{u} & =q & \text { in } \Omega, \\
u_{t} & =\mathrm{h}^{-1}(\mu) & \text { on } \Gamma,
\end{aligned}\right.
$$


which satisfies

$$
\|\mathbf{u}\|_{1} \leq C\left(\|q\|_{0}+\|\mu\|_{-\frac{1}{2}, \Gamma}\right),
$$

where $\mathrm{h}^{-1}(\cdot)$ is the inverse of the Riesz operator $\mathrm{h}: H_{00}^{\frac{1}{2}}(\Gamma) \rightarrow H_{*}^{-\frac{1}{2}}(\Gamma)$.

Firstly, let $q \in L_{0}^{2}(\Omega)$ then there exists $\hat{\mathbf{u}} \in \mathbf{H}_{0}^{1}(\Omega)$ such that (see, for example, [13])

$$
\operatorname{div} \hat{\mathbf{u}}=q, \quad \text { in } \Omega, \quad \text { and } \quad\|\hat{\mathbf{u}}\|_{1} \leq C_{1}\|q\|_{0} .
$$

with $C_{1}>0$ independent of $p$ and $\hat{\mathbf{u}}$.

Secondly, let $\mu \in L^{2}(\Gamma)$ and let $\psi$ be the unique solution to the following biharmonic problem:

$$
\left\{\begin{aligned}
\Delta^{2} \psi & =0 \text { in } \Omega, \\
\psi=0 & \text { on } \partial \Omega, \\
\frac{\partial \psi}{\partial n}=\chi & \text { on } \partial \Omega,
\end{aligned}\right.
$$

where $\chi$ is defined as the extension by zero of $\mathrm{h}^{-1}(\mu)$

$$
\chi= \begin{cases}0 & \text { on } \Gamma_{0}, \\ -\mathrm{h}^{-1}(\mu) & \text { on } \Gamma .\end{cases}
$$

Since $\mathrm{h}^{-1}(\mu) \in H_{00}^{\frac{1}{2}}(\Gamma)$, it is easy to see that $\chi \in H^{\frac{1}{2}}(\partial \Omega)$. From [20], since $\partial \Omega$ is regular enough ${ }^{3}$, it holds

$$
\psi \in H^{2}(\Omega) \quad \text { and } \quad\|\psi\|_{2} \leq C_{2}\|\chi\|_{\frac{1}{2}, \Gamma^{\Gamma}}
$$

where $C_{2}$ is a constant independent of $\psi$ and $\chi$.

Finally, we set $\mathbf{u}=\hat{\mathbf{u}}+\operatorname{curl} \psi$ with

$$
\operatorname{curl} \psi=\left[\begin{array}{c}
\frac{\partial \psi}{\partial x_{2}} \\
-\frac{\partial \psi}{\partial x_{1}}
\end{array}\right] .
$$

Hence, by the properties of $\hat{\mathbf{u}}$ and $\psi$, it comes that $\mathbf{u} \in \mathbf{V}(\Omega), u_{t}=\mathrm{h}^{-1}(\mu)$ on $\Gamma$ and $\operatorname{div} \mathbf{u}=q$ in $\Omega$, that is (4.6) holds.

Furthermore, from (4.8) and (4.10) we obtain:

$$
\begin{aligned}
\|\mathbf{u}\|_{1} & \leq\|\hat{\mathbf{u}}\|_{1}+\|\operatorname{curl} \psi\|_{1} \\
& \leq C_{1}\|q\|_{0}+\|\psi\|_{2} \\
& \leq C_{1}\|q\|_{0}+C_{2}\|\chi\|_{\frac{1}{2}, \Gamma} .
\end{aligned}
$$

Finally, using the continuity of $\mathrm{h}^{-1}$ in (4.11) we obtain (4.7) with $C=\max \left(C_{1}, C_{2} C_{3}\right)>0$ independent of $q$ and $\mu$ (here, $C_{3}$ stands for the continuity constant of $\mathrm{h}^{-1}$ ). Hence, the inf-sup condition is satisfied with $\alpha=(2 C)^{-1}$.

On the basis of the previous inf-sup condition (4.5) we have

\footnotetext{
${ }^{3}$ Following [21] a $\mathscr{C}^{1,1}$ class boundary (or a $\mathscr{C}^{1,1}$ curvilinear polygon boundary) would be enough, in the 2 dimensional case, to have the existence of a solution in $H^{2}$ to the biharmonic problem with non-homogeneous boundary conditions.
} 
Theorem 4.2. Under the hypothesis of Lemma 4.1, there exists a unique solution $(\mathbf{u},(p, \lambda))$ of the mixed problem (4.3). Moreover, $(\mathbf{u},(p, \lambda))$ is also the unique solution of the saddle-point problem (4.2).

Remark 4.3. Let $(\mathbf{u},(p, \lambda))$ be the solution of (4.3). Then

$$
\sigma_{t}=-\lambda
$$

\section{Finite Element approximation}

This section is devoted to present a finite element approximation of the saddle-point problem (4.2). The key point lies in a finite element discretization of the closed convex $\mathcal{Q}$ of the Lagrange multipliers which leads to a well-posed discrete problem and gives a good convergence rate for the approximate solution.

We use classical $P 1$ bubble- $P 1$ finite element to disretize $(\mathbf{u}, p)$ and $P 1$ finite element on $\Gamma$ for the Lagrange multiplier $\lambda$, see Section 5.1 below. This choice is motivated by the results presented in Arnold, Brezzi and Fortin [1] and Baillet and Sassi [3]. In Section 5.2, we establish the discrete inf-sup condition related to the discrete weak formulation of (4.3).

\subsection{Discretization}

The domain $\Omega \subset \mathbb{R}^{2}$ is supposed to be polygonal and convex. For the sake of simplicity, $\Gamma$ is assumed to be a straight line ${ }^{4}$. Let $\mathcal{T}_{h}$ be a regular partition of $\bar{\Omega}$ with triangles in the sense of [8]. We denote by $\mathbb{P}_{n}(\kappa)$ the space of polynomials of degree less or equal to $n \in \mathbb{N}$ defined on $\kappa \in \mathcal{T}_{h}$. We denote by $\mathcal{B}_{\kappa}$ the space of bubble functions defined on $\kappa$ which is a sub-space of $H_{0}^{1}(\kappa)$. Then we can define the following discrete spaces:

$$
\begin{aligned}
& \mathbb{B}=\bigoplus_{\kappa \in \mathcal{T}_{h}} \mathcal{B}_{\kappa}, \quad \mathcal{V}_{h}=\left\{\mathbf{v}_{h} \in \mathcal{C}^{0}(\bar{\Omega}) ; \mathbf{v}_{h \mid \kappa} \in \mathbb{P}_{1}(\kappa) \forall \kappa \in \mathcal{T}_{h}, \mathbf{v}_{h \mid \Gamma_{0}}=0, \text { and } \mathbf{v}_{h} \cdot \mathbf{n}_{\mid \Gamma}=0\right\}, \\
& \mathbf{V}_{h}=\left[\mathcal{V}_{h}+\mathbb{B}\right]^{2}, \quad \mathcal{W}_{h}=\left\{v_{h \mid \Gamma}, \quad v_{h} \in \mathcal{V}_{h}\right\}, \\
& \mathbb{L}_{h}=\left\{q_{h} \in \mathcal{C}^{0}(\bar{\Omega}) ; q_{h \mid \kappa} \in \mathbb{P}_{1}(\kappa) \forall \kappa \in \mathcal{T}_{h}, \int_{\Omega} q_{h} \mathrm{~d} \Omega=0\right\}, \\
& \mathcal{Q}_{h}=\left\{\mu_{h} \in \mathcal{W}_{h}, \int_{\Gamma} \mu_{h} \psi_{h} \mathrm{~d} \Gamma-\int_{\Gamma} g\left|\psi_{h}\right| \mathrm{d} \Gamma \leq 0 \forall \psi_{h} \in \mathcal{W}_{h}\right\}, \\
& \mathcal{M}_{h}=\mathbb{L}_{h} \times \mathcal{W}_{h}, \quad \Lambda_{h}=\mathbb{L}_{h} \times \mathcal{Q}_{h} .
\end{aligned}
$$

In what follows, we present some classical approximation results involving interpolation and projection operators between the continuous spaces and the above discrete spaces, that will be used in the error estimates. First, let $\mathcal{I}_{h}$ and $i_{h}$ be the Lagrange interpolation operators on $\mathbf{V}_{h}$ and $\mathcal{W}_{h}$ respectively. From [8], there exists a positive constant $C$ which does not depend on $h$ and such that for all $\mathbf{v} \in \mathbf{H}^{2}(\Omega)$ and for all $\psi \in H^{\frac{3}{2}}(\Gamma)$ it holds:

$$
\left\|\mathbf{v}-\mathcal{I}_{h} \mathbf{v}\right\|_{1} \leq C h\|\mathbf{v}\|_{2}, \quad\left\|\psi-i_{h} \psi\right\|_{0, \Gamma} \leq C h^{\frac{3}{2}}\|\psi\|_{\frac{3}{2}, \Gamma}
$$

Next, let $\Pi_{h}$ be the Clément's projection operator from $L^{2}(\Omega)$ on $\mathbb{L}_{h}$ introduced in [9]. The operator $\Pi_{h}$ verifies the following two properties:

$$
\Pi_{h} p \in \mathbb{L}_{h} \quad \text { and } \quad \int_{\Omega}\left(\Pi_{h} p-p\right) q_{h} \mathrm{~d} \Omega=0 \quad \forall q_{h} \in \mathbb{L}_{h} .
$$

\footnotetext{
${ }^{4}$ More general cases need higher technicalities, which are beyond the scope of this work.
} 
There exists a positive constant $C$ independent of $h$ such that for all $p \in H^{1}(\Omega) \cap L_{0}^{2}(\Omega)$

$$
\left\|p-\Pi_{h} p\right\|_{0} \leq C h\|p\|_{1}
$$

Now, we introduce the projection operator $\pi_{h}$ from $L^{2}(\Gamma)$ on $\mathcal{W}_{h}$ defined by:

$$
\pi_{h} \psi \in \mathcal{W}_{h}, \quad \int_{\Gamma}\left(\pi_{h} \psi-\psi\right) \mu_{h} \mathrm{~d} \Gamma=0 \quad \forall \mu_{h} \in \mathcal{W}_{h}
$$

The operator $\pi_{h}$ has the following approximation property: there exists a positive constant independent of $h$ such that for all $\psi \in H^{\frac{1}{2}+\tau}(\Gamma)$, for all $\tau \in[0,1]$ and any $\eta \in\left[0, \tau+\frac{1}{2}\right]$ one has (see $[4,11]$ ):

$$
h^{-\frac{1}{2}}\left\|\psi-\pi_{h} \psi\right\|_{-\frac{1}{2}, \Gamma}+h^{\eta}\left\|\psi-\pi_{h} \psi\right\|_{\eta, \Gamma} \leq C h^{\tau+\frac{1}{2}}\|\psi\|_{\frac{1}{2}+\tau, \Gamma}
$$

Finally, if $(\mathbf{u},(p, \lambda))$, the solution of (4.3), is such that $\mathbf{u} \in \mathbf{H}^{2}(\Omega)$, then the trace theorem and (4.12) imply

$$
\|\lambda\|_{\frac{1}{2}, \Gamma} \leq C\|\mathbf{u}\|_{2}
$$

Remark 5.1. Note that $\mathbf{V}_{h} \subset \mathbf{V}(\Omega)$ and $\mathcal{W}_{h} \subset \mathcal{W}$ while $\mathcal{Q}_{h}$ is an external approximation of $\mathcal{Q}, \mathcal{Q}_{h} \not \subset \mathcal{Q}$, so the discretization is non-conforming and would weaken its convergence rate.

Discretizing (4.3) we obtain

$$
\left\{\begin{array}{l}
\text { Find }\left(\mathbf{u}_{h},\left(p_{h}, \lambda_{h}\right)\right) \in \mathbf{V}_{h} \times \Lambda_{h} \text { such that: } \\
\qquad \begin{array}{rl}
a\left(\mathbf{u}_{h}, \mathbf{v}_{h}\right)+b\left(\left(p_{h}, \lambda_{h}\right), \mathbf{v}_{h}\right) & =L\left(\mathbf{v}_{h}\right) \\
b\left(\left(q_{h}-p_{h}, \mu_{h}-\lambda_{h}\right), \mathbf{u}_{h}\right) & \leq 0 \quad \forall\left(\mathbf{V}_{h},\right.
\end{array} \quad \forall\left(q_{h}\right) \in \Lambda_{h} .
\end{array}\right.
$$

\subsection{Discrete inf-sup condition}

First, we introduce an equivalent discrete formulation for (5.7) by eliminating the velocity with non-zero tangential component on $\Gamma$. Define

$$
\mathbf{V}_{h}^{0}=\left\{\mathbf{v}_{h} \in \mathbf{V}_{h},\left\langle\mu_{h}, v_{h t}\right\rangle=0 \quad \forall \mu_{h} \in \mathcal{W}_{h}\right\} .
$$

Then we can formulate problem (5.7) as

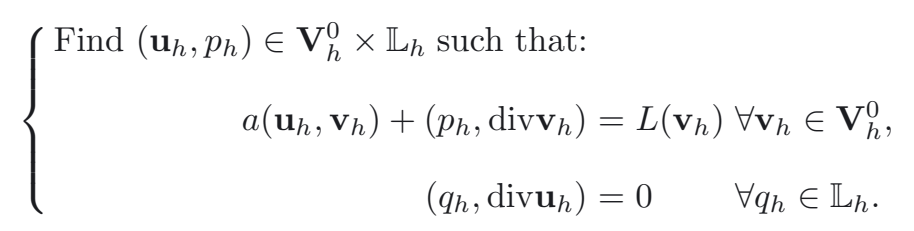

Now we define

$$
\mathbf{Z}_{h}=\left\{\mathbf{v}_{h} \in \mathbf{V}_{h},\left(q_{h}, \operatorname{div} \mathbf{v}_{h}\right)=0 \quad \forall q_{h} \in \mathbb{L}_{h}\right\} .
$$

Then we can also formulate problem (5.7) as

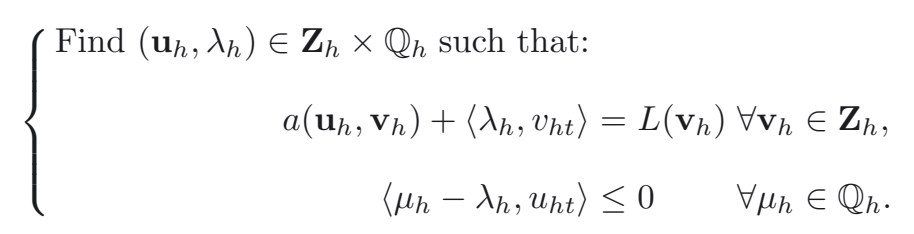


Note that $\left\{\mathbf{v}_{h} \in \mathbf{V}_{h},\left.\mathbf{v}_{h}\right|_{\partial \Omega}=0\right\} \subset \mathbf{V}_{h}^{0}$. From the usual discrete inf-sup condition for the Stokes problem (see for example $[13,20]$ ) we have the following inf-sup condition associated to problem (5.8).

Lemma 5.2. There is a constant $\beta_{1}>0$ independent of $h$ such that

$$
\sup _{\mathbf{v}_{h} \in \mathbf{V}_{h}^{0}} \frac{\left(q_{h}, \operatorname{div}_{h}\right)}{\left\|\mathbf{v}_{h}\right\|_{1}} \geq \beta_{1}\left\|q_{h}\right\|_{0} \quad \forall q_{h} \in \mathbb{L}_{h}
$$

Lemma 5.2, the coercivity of the bilinear form $a$ on $\mathbf{V}_{h}$ and the fact that $\mathbf{V}_{h}^{0} \subset \mathbf{V}_{h}$ guarantee the stability of problem (5.8)

To see that the discrete formulation (5.9) is stable, the next Lemma (proved in the Appendix) shows that the inf-sup condition between spaces $\mathbf{Z}_{h}$ and $\mathcal{W}_{h}$ holds.

Lemma 5.3. There is a constant $\beta_{2}>0$ independent of $h$ such that

$$
\sup _{\mathbf{v}_{h} \in \mathbf{Z}_{h}} \frac{\left\langle\mu_{h}, v_{h t}\right\rangle}{\left\|\mathbf{v}_{h}\right\|_{1}} \geq \beta_{2}\left\|\mu_{h}\right\|_{-\frac{1}{2}, \Gamma} \quad \forall \mu_{h} \in \mathcal{W}_{h}
$$

From Lemmas 5.2 and 5.3 we can show the following coupled discrete inf-sup condition

Proposition 5.4. There is a constant $\beta>0$ independent of $h$, such that

$$
\sup _{\mathbf{v}_{h} \in \mathbf{V}_{h}} \frac{b\left(\left(q_{h}, \mu_{h}\right), \mathbf{v}_{h}\right)}{\left\|\mathbf{v}_{h}\right\|_{1}} \geq \beta\left(\left\|q_{h}\right\|_{0}+\left\|\mu_{h}\right\|_{-\frac{1}{2}, \Gamma}\right) \quad \forall\left(q_{h}, \mu_{h}\right) \in \mathbb{L}_{h} \times \mathcal{W}_{h} .
$$

Proof. Given $\left(q_{h}, \mu_{h}\right) \in \mathbb{L}_{h} \times \mathcal{W}_{h}$, from Lemma 5.2 there exists $\hat{\mathbf{v}}_{h} \in \mathbf{V}_{h}^{0}$ such that

$$
\frac{\left(q_{h}, \operatorname{div} \hat{\mathbf{v}}_{h}\right)}{\left\|\hat{\mathbf{v}}_{h}\right\|_{1}} \geq \beta_{1}\left\|q_{h}\right\|_{0}
$$

From Lemma 5.3 there exists $\mathbf{z}_{h} \in \mathbf{Z}_{h}$ such that

$$
\frac{\left\langle\mu_{h}, z_{h t}\right\rangle}{\left\|z_{h}\right\|_{1}} \geq \beta_{2}\left\|\mu_{h}\right\|_{-\frac{1}{2}, \Gamma} .
$$

Observe that

$$
\sup _{\mathbf{v}_{h} \in \mathbf{V}_{h}} \frac{b\left(\left(q_{h}, \mu_{h}\right), \mathbf{v}_{h}\right)}{\left\|\mathbf{v}_{h}\right\|_{1}} \geq \frac{b\left(\left(q_{h}, \mu_{h}\right), \hat{\mathbf{v}}_{h}\right)}{\left\|\hat{\mathbf{v}}_{h}\right\|_{1}}=\frac{\left(q_{h}, \operatorname{div} \hat{\mathbf{v}}_{h}\right)}{\left\|\hat{\mathbf{v}}_{h}\right\|_{1}} \geq \beta_{1}\left\|q_{h}\right\|_{0} .
$$

Analogously

$$
\sup _{\mathbf{v}_{h} \in \mathbf{V}_{h}} \frac{b\left(\left(q_{h}, \mu_{h}\right), \mathbf{v}_{h}\right)}{\left\|\mathbf{v}_{h}\right\|_{1}} \geq \frac{\left\langle\mu_{h}, z_{h t}\right\rangle}{\left\|\mathbf{z}_{h}\right\|_{1}} \geq \beta_{2}\left\|\mu_{h}\right\|_{-\frac{1}{2}, \Gamma}
$$

Then

$$
\sup _{\mathbf{v}_{h} \in \mathbf{V}_{h}} \frac{b\left(\left(q_{h}, \mu_{h}\right), \mathbf{v}_{h}\right)}{\left\|\mathbf{v}_{h}\right\|_{1}} \geq \frac{\min \left(\beta_{1}, \beta_{2}\right)}{2}\left(\left\|q_{h}\right\|_{0}+\left\|\mu_{h}\right\|_{-\frac{1}{2}, \Gamma}\right) .
$$


The inf-sup condition (5.12), together with the fact that $a$ is $\mathbf{V}_{h}$-elliptic guarantees the existence and uniqueness of a solution to $(5.7)$ (see $[7,20])$.

\section{ERror estimates}

In this section we will establish a priori error estimates for the mixed finite element approximation (5.7). To this end, we note that Lemma 4.1, and therefore Theorem 4.2, is still valid in the case of a polygonal domain in which $\Gamma$ is supposed to be a straight line ${ }^{5}$, that is, we have existence and uniqueness of a solution to problem (4.3) for this boundary regularity. We start with the following lemma inspired of $[3,23]$.

Lemma 6.1. Let $(\mathbf{u}, p, \lambda)$ and $\left(\mathbf{u}_{h}, p_{h}, \lambda_{h}\right)$ be solutions to (4.3) and (5.7), respectively. Then for any $\left(\mathbf{v}_{h}, q_{h}, \mu_{h}\right) \in \mathbf{V}_{h} \times \Lambda_{h}$ it holds:

$$
\begin{aligned}
a\left(\mathbf{u}-\mathbf{u}_{h}, \mathbf{u}-\mathbf{u}_{h}\right) \leq & a\left(\mathbf{u}-\mathbf{u}_{h}, \mathbf{u}-\mathbf{v}_{h}\right)+b\left((p, \lambda)-\left(q_{h}, \mu_{h}\right), \mathbf{u}_{h}-\mathbf{u}\right)+b\left((p, \lambda)-\left(p_{h}, \lambda_{h}\right), \mathbf{u}-\mathbf{v}_{h}\right) \\
& +b\left((p, \lambda)-\left(q_{h}, \mu_{h}\right), \mathbf{u}\right)+b\left(\left(p_{h}, \lambda_{h}\right)-(p, \lambda), \mathbf{u}\right) .
\end{aligned}
$$

Proof. Let $\mathbf{v}_{h}$ be an element of $\mathbf{V}_{h}$. It follows that:

$$
a\left(\mathbf{u}-\mathbf{u}_{h}, \mathbf{u}-\mathbf{u}_{h}\right)=a\left(\mathbf{u}-\mathbf{u}_{h}, \mathbf{u}-\mathbf{v}_{h}\right)+a\left(\mathbf{u}-\mathbf{u}_{h}, \mathbf{v}_{h}-\mathbf{u}_{h}\right) .
$$

Using the first equations of (4.3) and of (5.7), we obtain:

$$
\begin{aligned}
a\left(\mathbf{u}-\mathbf{u}_{h}, \mathbf{v}_{h}-\mathbf{u}_{h}\right) & =a\left(\mathbf{u}, \mathbf{v}_{h}-\mathbf{u}_{h}\right)-a\left(\mathbf{u}_{h}, \mathbf{v}_{h}-\mathbf{u}_{h}\right), \\
& =L\left(\mathbf{v}_{h}-\mathbf{u}_{h}\right)-b\left((p, \lambda), \mathbf{v}_{h}-\mathbf{u}_{h}\right)-L\left(\mathbf{v}_{h}-\mathbf{u}_{h}\right)+b\left(\left(p_{h}, \lambda_{h}\right), \mathbf{v}_{h}-\mathbf{u}_{h}\right), \\
& =b\left((p, \lambda), \mathbf{u}_{h}-\mathbf{v}_{h}\right)+b\left(\left(p_{h}, \lambda_{h}\right), \mathbf{v}_{h}-\mathbf{u}_{h}\right) .
\end{aligned}
$$

Then we deduce

$$
a\left(\mathbf{u}-\mathbf{u}_{h}, \mathbf{u}-\mathbf{u}_{h}\right)=a\left(\mathbf{u}-\mathbf{u}_{h}, \mathbf{u}-\mathbf{v}_{h}\right)+b\left((p, \lambda), \mathbf{u}_{h}-\mathbf{v}_{h}\right)+b\left(\left(p_{h}, \lambda_{h}\right), \mathbf{v}_{h}-\mathbf{u}_{h}\right) .
$$

Finally we have

$$
\begin{aligned}
a\left(\mathbf{u}-\mathbf{u}_{h}, \mathbf{u}-\mathbf{u}_{h}\right)= & a\left(\mathbf{u}-\mathbf{u}_{h}, \mathbf{u}-\mathbf{v}_{h}\right)+b\left((p, \lambda)-\left(q_{h}, \mu_{h}\right), \mathbf{u}_{h}-\mathbf{u}\right)+b\left((p, \lambda)-\left(p_{h}, \lambda_{h}\right), \mathbf{u}-\mathbf{v}_{h}\right) \\
& +b\left((p, \lambda)-\left(q_{h}, \mu_{h}\right), \mathbf{u}\right)+b\left(\left(p_{h}, \lambda_{h}\right)-(p, \lambda), \mathbf{u}\right) \\
& +b\left(\left(q_{h}, \mu_{h}\right)-\left(p_{h}, \lambda_{h}\right), \mathbf{u}_{h}\right) .
\end{aligned}
$$

The inequality in (5.7) implies that $b\left(\left(q_{h}, \mu_{h}\right)-\left(p_{h}, \lambda_{h}\right), \mathbf{u}_{h}\right) \leq 0$ for all $\left(q_{h}, \mu_{h}\right) \in \Lambda_{h}$, and then, the error estimate (6.13) follows.

By deriving an upper bound of the terms involved in the right hand side of (6.13), for a particular $\left(\mathbf{v}_{h}, p_{h}, \mu_{h}\right)$, we will prove the following

\footnotetext{
${ }^{5}$ In this case we can verify that the boundary data in the biharmonic problem (4.9) satisfy the compatibility condition in ([19], Thm. 3 p. 359), since the tangential vector on $\Gamma$ is now a constant vector. Hence, the boundary data can be lifted by a $H^{2}(\Omega)$ function.
} 
Lemma 6.2. Let $(\mathbf{u}, p, \lambda),\left(\mathbf{u}_{h}, p_{h}, \lambda_{h}\right)$ be the solution to (4.3) and (5.7), respectively. Suppose that $\mathbf{u} \in \mathbf{H}^{2}(\Omega)$ and $p \in H^{1}(\Omega)$. Then

$$
\left\|\mathbf{u}-\mathbf{u}_{h}\right\|_{1}^{2} \leq C\left(\left(h^{\frac{3}{2}}+h\left\|\lambda-\lambda_{h}\right\|_{-\frac{1}{2}, \Gamma}+h\left\|p-p_{h}\right\|_{0}\right),\right.
$$

where $C$ is a positive constant depending only on $\|\mathbf{u}\|_{2},\|p\|_{1}$ and $\|g\|_{0, \Gamma}$.

Proof. We will show that there exists $\left(\mathbf{v}_{h},\left(q_{h}, \mu_{h}\right)\right) \in \mathbf{V}_{h} \times \Lambda_{h}$ satisfying:

$$
\begin{cases}a\left(\mathbf{u}-\mathbf{u}_{h}, \mathbf{u}-\mathbf{v}_{h}\right) & \leq C(\mathbf{u}) h\left\|\mathbf{u}-\mathbf{u}_{h}\right\|_{1}, \\ b\left((p, \lambda)-\left(q_{h}, \mu_{h}\right), \mathbf{u}_{h}-\mathbf{u}\right) \leq C(\mathbf{u}, p) h\left\|\mathbf{u}-\mathbf{u}_{h}\right\|_{1}, \\ b\left(\left(p_{h}, \lambda_{h}\right)-(p, \lambda), \mathbf{u}-\mathbf{v}_{h}\right) \leq C h\left\{\left\|\mathbf{u}-\mathbf{u}_{h}\right\|_{1}+C(p) h+C(\mathbf{u}) h\right\}, \\ b\left((p, \lambda)-\left(q_{h}, \mu_{h}\right), \mathbf{u}\right) \quad \leq C(\mathbf{u})^{2} h^{2}, \\ b\left(\left(p_{h}, \lambda_{h}\right)-(p, \lambda), \mathbf{u}\right) \leq C(\mathbf{u})\left(h\left\|\lambda-\lambda_{h}\right\|_{-\frac{1}{2}, \Gamma}+C(\mathbf{u}) h^{\frac{3}{2}}+C(g) h^{\frac{3}{2}}\right) .\end{cases}
$$

In what follows we choose $\mathbf{v}_{h}=\mathcal{I}_{h} \mathbf{u}, q_{h}=\Pi_{h} p$ and $\mu_{h}=\pi_{h} \lambda$. From the definition of $\mathcal{Q}, \mathcal{Q}_{h}$ and (5.4), it is readily seen that $\pi_{h} \lambda \in \mathcal{Q}_{h}$ :

$$
\forall \psi_{h} \in \mathcal{W}_{h} \quad \int_{\Gamma}\left(\pi_{h} \lambda\right) \psi_{h} \mathrm{~d} \Gamma=\int_{\Gamma} \lambda \psi_{h} \mathrm{~d} \Gamma \leq \int_{\Gamma} g \psi_{h} \mathrm{~d} \Gamma \leq \int_{\Gamma} g\left|\psi_{h}\right| \mathrm{d} \Gamma
$$

(i) The first term of (6.15) is obtained by using the continuity of $a(\cdot, \cdot)$ and the property $(5.1)$

$$
\begin{aligned}
a\left(\mathbf{u}-\mathbf{u}_{h}, \mathbf{u}-\mathbf{v}_{h}\right) & \leq C\left\|\mathbf{u}-\mathbf{u}_{h}\right\|_{1}\left\|\mathbf{u}-\mathbf{v}_{h}\right\|_{1} \\
& \leq C(\mathbf{u}) h\left\|\mathbf{u}-\mathbf{u}_{h}\right\|_{1} .
\end{aligned}
$$

(ii) Using (5.3) and (5.5) we obtain the second inequality of (6.15):

$$
\begin{aligned}
b\left((p, \lambda)-\left(q_{h}, \mu_{h}\right), \mathbf{u}_{h}-\mathbf{u}\right) & =-\left(p-q_{h}, \operatorname{div}\left(\mathbf{u}_{h}-\mathbf{u}\right)\right)+\left\langle\lambda-\mu_{h}, u_{h t}-u_{t}\right\rangle \\
& \leq C\left\{\left\|p-q_{h}\right\|_{0}+\left\|\lambda-\mu_{h}\right\|_{-\frac{1}{2}, \Gamma}\right\}\left\|\mathbf{u}-\mathbf{u}_{h}\right\|_{1} \\
& \leq C(\mathbf{u}, p) h\left\|\mathbf{u}-\mathbf{u}_{h}\right\|_{1} .
\end{aligned}
$$

(iii) Further, using again (5.1), there holds that

$$
\begin{aligned}
b\left((p, \lambda)-\left(p_{h}, \lambda_{h}\right), \mathbf{u}-\mathbf{v}_{h}\right) & =-\left(p-p_{h}, \operatorname{div}\left(\mathbf{u}-\mathbf{v}_{h}\right)\right)+\left\langle\lambda-\lambda_{h}, u_{t}-v_{h t}\right\rangle, \\
& \leq C\left\{\left\|p-p_{h}\right\|_{0}+\left\|\lambda-\lambda_{h}\right\|_{-\frac{1}{2}, \Gamma}\right\}\left\|\mathbf{u}-\mathbf{v}_{h}\right\|_{1} \\
& \leq C h\left\{\left\|p-p_{h}\right\|_{0}+\left\|\lambda-\lambda_{h}\right\|_{-\frac{1}{2}, \Gamma}\right\} .
\end{aligned}
$$


(iv) To estimate the fourth term of (6.15) we invoke the definition of the $L^{2}$-projection operator on $\Gamma(5.4)$ and the approximation property (5.5):

$$
\begin{aligned}
b\left((p, \lambda)-\left(q_{h}, \mu_{h}\right), \mathbf{u}\right) & =\int_{\Gamma}\left(\lambda-\mu_{h}\right) u_{t} \mathrm{~d} \Gamma \\
& =\int_{\Gamma}\left(\lambda-\pi_{h} \lambda\right) u_{t} \mathrm{~d} \Gamma \\
& =\int_{\Gamma}\left(\lambda-\pi_{h} \lambda\right) u_{t} \mathrm{~d} \Gamma-\int_{\Gamma}\left(\lambda-\pi_{h} \lambda\right) \pi_{h} u_{t} \mathrm{~d} \Gamma, \\
& =\int_{\Gamma}\left(\lambda-\pi_{h} \lambda\right)\left(u_{t}-\pi_{h} u_{t}\right) \mathrm{d} \Gamma, \\
& \leq\left\|\lambda-\pi_{h} \lambda\right\|_{0, \Gamma}\left\|u_{t}-\pi_{h} u_{t}\right\|_{0, \Gamma}, \\
& \leq C h^{2} .
\end{aligned}
$$

(v) Now we shall estimate the fifth term of (6.15). Noticing that, since $\lambda_{h} \in Q_{h}$ then

$$
\int_{\Gamma}\left(\lambda_{h} i_{h}\left(u_{t}\right)-g\left|i_{h}\left(u_{t}\right)\right|\right) \mathrm{d} \Gamma \leq 0
$$

and that, from (3.2) and (4.12), we have

$$
\lambda u_{t}-g\left|u_{t}\right|=0 \quad \text { a.e. on } \Gamma .
$$

Therefore, combining this with (5.1) and (5.6), we have

$$
\begin{aligned}
b\left(\left(p_{h}, \lambda_{h}\right)-(p, \lambda), \mathbf{u}\right) & =\int_{\Gamma}\left(\lambda_{h}-\lambda\right) u_{t} \mathrm{~d} \Gamma \\
& =\int_{\Gamma}\left(\lambda_{h}-\lambda\right)\left(u_{t}-i_{h}\left(u_{t}\right)\right) \mathrm{d} \Gamma+\int_{\Gamma}\left(\lambda_{h}-\lambda\right) i_{h}\left(u_{t}\right) \mathrm{d} \Gamma+\int_{\Gamma}\left(\lambda u_{t}-g\left|u_{t}\right|\right) \mathrm{d} \Gamma \\
& =\int_{\Gamma}\left(\lambda_{h}-\lambda\right)\left(u_{t}-i_{h}\left(u_{t}\right)\right) \mathrm{d} \Gamma+\int_{\Gamma}\left(\lambda_{h} i_{h}\left(u_{t}\right)-g\left|u_{t}\right|\right) \mathrm{d} \Gamma+\int_{\Gamma} \lambda\left(u_{t}-i_{h}\left(u_{t}\right)\right) \mathrm{d} \Gamma, \\
& \leq \int_{\Gamma}\left(\lambda_{h}-\lambda\right)\left(u_{t}-i_{h}\left(u_{t}\right)\right) \mathrm{d} \Gamma+\int_{\Gamma} g\left(\left|i_{h}\left(u_{t}\right)\right|-\left|u_{t}\right|\right) \mathrm{d} \Gamma+\int_{\Gamma} \lambda\left(u_{t}-i_{h}\left(u_{t}\right)\right) \mathrm{d} \Gamma, \\
& \leq \int_{\Gamma}\left(\lambda_{h}-\lambda\right)\left(u_{t}-i_{h}\left(u_{t}\right)\right) \mathrm{d} \Gamma+\int_{\Gamma} g\left|i_{h}\left(u_{t}\right)-u_{t}\right| \mathrm{d} \Gamma+\int_{\Gamma} \lambda\left(u_{t}-i_{h}\left(u_{t}\right)\right) \mathrm{d} \Gamma, \\
& \leq\left\|\lambda_{h}-\lambda\right\|\left\|_{-\frac{1}{2}, \Gamma}\right\| u_{t}-i_{h}\left(u_{t}\right)\left\|_{\frac{1}{2}, \Gamma}+\right\| g\left\|_{0, \Gamma}\right\| u_{t}-i_{h}\left(u_{t}\right)\left\|_{0, \Gamma}+\right\| \lambda\left\|_{0, \Gamma}\right\| u_{t}-i_{h}\left(u_{t}\right) \|_{0, \Gamma}, \\
& \leq C(\mathbf{u}) h\left\|\lambda-\lambda_{h}\right\|_{-\frac{1}{2}, \Gamma}+C(\mathbf{u})^{2} h^{\frac{3}{2}}+C(g) C(\mathbf{u}) h^{\frac{3}{2}}, \\
& \leq C\left\{h\left\|\lambda-\lambda_{h}\right\|_{-\frac{1}{2}, \Gamma}+h^{\frac{3}{2}}\right\} .
\end{aligned}
$$

Assembling the estimates (i)-(v) and using Lemma 6.1 and the $\mathbf{V}$-ellipticity of the bilinear form $a(\cdot, \cdot)$, we finally arrive at the following estimate

$$
\left\|\mathbf{u}-\mathbf{u}_{h}\right\|_{1}^{2} \leq C\left(h\left\|\lambda-\lambda_{h}\right\|_{-\frac{1}{2}, \Gamma}+h\left\|\mathbf{u}-\mathbf{u}_{h}\right\|_{1}+h\left\|p-p_{h}\right\|_{0}\right)+C h^{\frac{3}{2}}
$$


then, using the Young's inequality, we can write for every constant $A>0$

$$
C h\left\|\mathbf{u}-\mathbf{u}_{h}\right\|_{1} \leq C\left(A h^{2}+\frac{1}{A}\left\|\mathbf{u}-\mathbf{u}_{h}\right\|_{1}^{2}\right) .
$$

Finally, if we take $A$ such that $\frac{C}{A}<1$, then it leads to the desired result.

The next two lemmas will give us error estimates for the pressure and the Lagrange multiplier $\lambda$ in terms of $h$ and the velocity error.

Lemma 6.3. Let $(\mathbf{u}, p, \lambda)$ and $\left(\mathbf{u}_{h}, p_{h}, \lambda_{h}\right)$ be solutions to (4.3) and (5.7), respectively. Suppose that $\mathbf{u} \in \mathbf{H}^{2}(\Omega)$ and $p \in H^{1}(\Omega)$. Then

$$
\left\|p-p_{h}\right\|_{0} \leq C\left\{h+\left\|\mathbf{u}-\mathbf{u}_{h}\right\|_{1}\right\},
$$

where $C$ is a positive constant depending only on $\|\mathbf{u}\|_{2}$ and $\|p\|_{1}$.

Proof. Since $(\mathbf{u}, p, \lambda)$ and $\left(\mathbf{u}_{h}, p_{h}, \lambda_{h}\right)$ are solutions to (4.3) and (5.7), respectively, then for $\mathbf{v}_{h} \in \mathbf{V}_{h}$

$$
\begin{array}{r}
a\left(\mathbf{u}, \mathbf{v}_{h}\right)-\left(p, \operatorname{div}\left(\mathbf{v}_{h}\right)\right)+\left\langle\lambda, v_{t h}\right\rangle=L\left(\mathbf{v}_{h}\right), \\
a\left(\mathbf{u}_{h}, \mathbf{v}_{h}\right)-\left(p_{h}, \operatorname{div}\left(\mathbf{v}_{h}\right)\right)+\left\langle\lambda_{h}, v_{t h}\right\rangle=L\left(\mathbf{v}_{h}\right),
\end{array}
$$

by subtracting these two equations we obtain

$$
a\left(\mathbf{u}-\mathbf{u}_{h}, \mathbf{v}_{h}\right)-\left(p-p_{h}, \operatorname{div}\left(\mathbf{v}_{h}\right)\right)+\left\langle\lambda-\lambda_{h}, v_{t h}\right\rangle=0 \quad \forall \mathbf{v}_{h} \in \mathbf{V}_{h},
$$

and taking $\mathbf{v}_{h} \in \mathbf{V}_{h}^{0},(6.20)$ becomes

$$
a\left(\mathbf{u}-\mathbf{u}_{h}, \mathbf{v}_{h}\right)+\left(p-p_{h}, \operatorname{div}\left(\mathbf{v}_{h}\right)\right)=0 \quad \forall \mathbf{v}_{h} \in \mathbf{V}_{h}^{0} .
$$

Therefore

$$
\left(p_{h}-\Pi_{h} p, \operatorname{div}\left(\mathbf{v}_{h}\right)\right)=\left(p_{h}-p, \operatorname{div}\left(\mathbf{v}_{h}\right)\right)+\left(p-\Pi_{h} p, \operatorname{div}\left(\mathbf{v}_{h}\right)\right)=a\left(\mathbf{u}-\mathbf{u}_{h}, \mathbf{v}_{h}\right)+\left(p-\Pi_{h} p, \operatorname{div}\left(\mathbf{v}_{h}\right)\right),
$$

and, using (5.3),

$$
\left(p_{h}-\Pi_{h} p, \operatorname{div}\left(\mathbf{v}_{h}\right)\right) \leq C\left\|\mathbf{u}-\mathbf{u}_{h}\right\|_{1}\left\|\mathbf{v}_{h}\right\|_{1}+C h\|p\|_{1}\left\|\mathbf{v}_{h}\right\|_{1} \quad \forall \mathbf{v}_{h} \in \mathbf{V}_{h}^{0}
$$

The inf-sup condition (5.10) yields to

$$
\beta_{1}\left\|p_{h}-\Pi_{h} p\right\|_{0} \leq \sup _{\mathbf{v}_{h} \in \mathbf{V}_{h}^{0}} \frac{\left(p_{h}-\Pi_{h} p, \operatorname{div}\left(\mathbf{v}_{h}\right)\right)}{\left\|\mathbf{v}_{h}\right\|_{1}} \leq C\left\|\mathbf{u}-\mathbf{u}_{h}\right\|_{1}+C h\|p\|_{1} .
$$

The triangle inequality

$$
\left\|p-p_{h}\right\|_{0} \leq\left\|p-\Pi_{h} p\right\|_{0}+\left\|\Pi_{h} p-p_{h}\right\|_{0}
$$

together with (6.21) and (5.3) proves (6.19).

Lemma 6.4. Let $(\mathbf{u}, p, \lambda)$ and $\left(\mathbf{u}_{h}, p_{h}, \lambda_{h}\right)$ be solutions to (4.3) and (5.7) respectively. Suppose that $\mathbf{u} \in \mathbf{H}^{2}(\Omega)$ and $p \in H^{1}(\Omega)$. Then

$$
\left\|\lambda-\lambda_{h}\right\|_{-\frac{1}{2}, \Gamma} \leq C\left\{h+\left\|\mathbf{u}-\mathbf{u}_{h}\right\|_{1}\right\}
$$

where $C$ is a positive constant depending only on $\|\mathbf{u}\|_{2}$. 
Proof. Introducing $\pi_{h} \lambda$ in (6.20) we have:

$$
\left\langle\pi_{h} \lambda-\lambda_{h}, v_{h t}\right\rangle=a\left(\mathbf{u}_{h}-\mathbf{u}, \mathbf{v}_{h}\right)+\left(p-p_{h}, \operatorname{div}\left(\mathbf{v}_{h}\right)\right)-\left\langle\lambda-\pi_{h} \lambda, v_{h t}\right\rangle \quad \forall \mathbf{v}_{h} \in \mathbf{V}_{h}
$$

Therefore

$$
\left\langle\pi_{h} \lambda-\lambda_{h}, v_{h t}\right\rangle \leq C\left\|\mathbf{u}-\mathbf{u}_{h}\right\|_{1}\left\|\mathbf{v}_{h}\right\|_{1}+C\left\|p-p_{h}\right\|\left\|_{0}\right\| \mathbf{v}_{h}\left\|_{1}+C h\right\| \lambda\left\|_{\frac{1}{2}, \Gamma}\right\| \mathbf{v}_{h} \|_{1},
$$

for all $\mathbf{v}_{h} \in \mathbf{V}_{h}$, and in particular for all $\mathbf{v}_{h} \in \mathbf{Z}_{h}$. Using the inf-sup condition (5.11) combined with (6.19) we obtain

$$
\begin{aligned}
\beta_{2}\left\|\pi_{h} \lambda-\lambda_{h}\right\|_{-\frac{1}{2}, \Gamma} & \leq \sup _{\mathbf{v}_{h} \in \mathbf{Z}_{h}} \frac{\left\langle\pi_{h} \lambda-\lambda_{h}, v_{h t}\right\rangle}{\left\|\mathbf{v}_{h}\right\|_{1}} \\
& \leq C\left(\left\|\mathbf{u}-\mathbf{u}_{h}\right\|_{1}+\left\|p-p_{h}\right\|_{0}+h\right) \\
& \leq C\left(\left\|\mathbf{u}-\mathbf{u}_{h}\right\|_{1}+h\right)
\end{aligned}
$$

Inserting the estimates (5.5) and (6.23) in the triangle inequality

$$
\left\|\lambda-\lambda_{h}\right\|_{-\frac{1}{2}, \Gamma} \leq\left\|\lambda-\pi_{h} \lambda\right\|_{-\frac{1}{2}, \Gamma}+\left\|\pi_{h} \lambda-\lambda_{h}\right\|_{-\frac{1}{2}, \Gamma}
$$

we finally arrive at the estimate (6.22).

Using Lemmas 6.2, 6.3 and 6.4, we are now able to prove our main a priori error estimate result:

Theorem 6.5. Let $(\mathbf{u}, p, \lambda)$ and $\left(\mathbf{u}_{h}, p_{h}, \lambda_{h}\right)$ be solutions to (4.3) and (5.7) respectively. Suppose that $\mathbf{u} \in \mathbf{H}^{2}(\Omega)$ and $p \in H^{1}(\Omega)$. Then

$$
\left\|\mathbf{u}-\mathbf{u}_{h}\right\|_{1}+\left\|p-p_{h}\right\|_{0}+\left\|\lambda-\lambda_{h}\right\|_{-\frac{1}{2}, \Gamma} \leq C h^{\frac{3}{4}},
$$

where $C$ is a positive constant depending only on $\|\mathbf{u}\|_{2},\|p\|_{1}$ and $\|g\|_{0, \Gamma}$.

Proof. By using (6.19) and (6.22) in (6.14), we can write:

$$
\begin{aligned}
\left\|\mathbf{u}-\mathbf{u}_{h}\right\|_{1}^{2} & \leq C\left\{h\left\|\lambda-\lambda_{h}\right\|_{-\frac{1}{2}, \Gamma}+h\left\|p-p_{h}\right\|_{0}+h^{\frac{3}{2}}\right\}, \\
& \leq C h\left\{h+\left\|\mathbf{u}-\mathbf{u}_{h}\right\|_{1}\right\}+C h^{\frac{3}{2}} \\
& \leq C h^{2}+C h\left\|\mathbf{u}-\mathbf{u}_{h}\right\|_{1}+C h^{\frac{3}{2}},
\end{aligned}
$$

hence, using again Young's inequality,

$$
\left\|\mathbf{u}-\mathbf{u}_{h}\right\|_{1} \leq C h^{\frac{3}{4}}
$$

Consequently, (6.19) and (6.22) become

$$
\left\|p-p_{h}\right\|_{0} \leq C h^{\frac{3}{4}} \quad \text { and } \quad\left\|\lambda-\lambda_{h}\right\|_{-\frac{1}{2}, \Gamma} \leq C h^{\frac{3}{4}},
$$

which leads to the desired result.

Remark 6.6. We recall that a $\mathbf{H}^{2} \times H^{1}$ regularity result for the Stokes problem with slip boundary conditions is obtained under the hypotheses of Theorem 3.2 (see [30,31]). When $\Gamma$ and $\Gamma_{D}$ are not connected components and - or - have less regularity (e.g. only Lipschitz continuous boundaries), an analogous regularity result is, as far as we known, an open problem. 


\section{Conclusion}

A three field mixed formulation of the Stokes problem with Tresca boundary conditions has been introduced and studied. The convergence analysis and a priori error estimates of the discrete corresponding problem have been established. In particular, we show an optimal error estimate of order $h^{\frac{3}{4}}$ for the velocity when it is approximated by classical $P 1$ bubble finite element. The implementation of the mixed finite element formulation proposed in Section 5 will be presented in a forthcoming paper.

\section{Appendix A}

Proof of Lemma 5.3. The proof presented here follows the same ideas as in Proposition 3.3 from [10]. The main difference is that we are working with the space $\mathbf{Z}_{h}$ and with a Stokes extension operator $\mathbf{R}_{h}$.

Let $\mu_{h} \in \mathcal{W}_{h}$. There is $\psi \in H^{\frac{1}{2}}(\Gamma)$ with $\|\psi\|_{\frac{1}{2}, \Gamma}=1$, so that

$$
\left\langle\mu_{h}, \psi\right\rangle=\int_{\Gamma} \mu_{h} \psi \mathrm{d} \Gamma=\left\|\mu_{h}\right\|_{-\frac{1}{2}, \Gamma} .
$$

The definition of $\pi_{h}$ in (5.4) leads to

$$
\int_{\Gamma} \mu_{h} \psi \mathrm{d} \Gamma=\int_{\Gamma} \mu_{h} \pi_{h} \psi \mathrm{d} \Gamma
$$

For any $\psi_{h} \in \mathcal{W}_{h}$, define $\mathbf{R}_{h}\left(\psi_{h}\right) \in \mathbf{V}_{h}$ as the discrete velocity solution of the following problem (the discrete Stokes extension):

$$
\left\{\begin{aligned}
a\left(\mathbf{R}_{h}\left(\psi_{h}\right), \mathbf{v}_{h}\right)+\left(p_{h}, \operatorname{div} \mathbf{v}_{h}\right)=0 & \forall \mathbf{v}_{h} \in \mathbf{V}_{h}, \text { such that } v_{h t}=0 \text { on } \Gamma, \\
\left(q_{h}, \operatorname{div} \mathbf{R}_{h}\left(\psi_{h}\right)\right)=0 & \forall q_{h} \in \mathbb{L}_{h}, \\
\left(\mathbf{R}_{h}\left(\psi_{h}\right)\right) . \mathbf{t}=\psi_{h} & \text { on } \Gamma .
\end{aligned}\right.
$$

Therefore using [28], we have

$$
\left\|\mathbf{R}_{h}\left(\psi_{h}\right)\right\|_{1} \leq C_{1}\left\|\psi_{h}\right\|_{\frac{1}{2}, \Gamma}
$$

Take $\mu_{h} \in \mathcal{W}_{h}$ and let $\mathbf{w}_{h}=\mathbf{R}_{h}\left(\pi_{h} \psi\right) \in \mathbf{V}_{h}$ be the velocity solution of (A.1). Then $\mathbf{w}_{h} \in \mathbf{Z}_{h}$ and

$$
\left\|\mathbf{w}_{h}\right\|_{1} \leq C_{1}\left\|\pi_{h} \psi\right\|_{\frac{1}{2}, \Gamma} \leq C_{2}\|\psi\|_{\frac{1}{2}, \Gamma} .
$$

Taking into account that $\|\psi\|_{\frac{1}{2}, \Gamma}=1$, we finally come to the conclusion that there is a positive constant $C_{2}$ independent of $h$ (and of $\mu_{h}$ ) such that

$$
\begin{aligned}
\frac{1}{C_{2}}\left\|\mu_{h}\right\|_{-\frac{1}{2}, \Gamma} \leq \frac{\left\|\mu_{h}\right\|_{-\frac{1}{2}, \Gamma}}{\left\|\mathbf{w}_{h}\right\|_{1}} & =\frac{\int_{\Gamma} \mu_{h} w_{h t} \mathrm{~d} \Gamma}{\left\|\mathbf{w}_{h}\right\|_{1}} \\
& =\frac{\left\langle\mu_{h}, w_{h t}\right\rangle}{\left\|\mathbf{w}_{h}\right\|_{1}} \\
& \leq \sup _{\mathbf{v}_{h} \in \mathbf{Z}_{h}} \frac{\left\langle\mu_{h}, v_{h t}\right\rangle}{\left\|\mathbf{v}_{h}\right\|_{1}} .
\end{aligned}
$$

Then the inf-sup condition (5.11) is proved to be true. 


\section{REFERENCES}

[1] D. Arnold, F. Brezzi and M. Fortin, A stable finite element for the Stokes equations. Calcolo 21 (1984) $337-344$.

[2] M. Ayadi, M. K. Gdoura and T. Sassi, Mixed formulation for Stokes problem with Tresca friction. C. R. Acad. Sci. Paris, Ser. I 348 (2010) 1069-1072.

[3] L. Baillet and T. Sassi, Mixed finite element methods for the Signorini problem with friction. Numer. Methods Partial Differ. Eq. 22 (2006) 1489-1508.

[4] F. Ben Belgacem and Y. Renard, Hybrid finite element method for the Signorini problem. Math. Comput. 72 (2003) $1117-1145$.

[5] M. Boukrouche and F. Saidi, Non-isothermal lubrication problem with Tresca fluid-solid interface law. Part I. Nonlinear Analysis: Real World Appl. 7 (2006) 1145-1166.

[6] F. Brezzi, W. Hager and P.A. Raviart, Error estimates for the finite element solution of variational inequalities, part II. Mixed methods. Numer. Math. 31 (1978) 1-16.

[7] F. Brezzi and M. Fortin, Mixed and Hybrid Finite Element Methods, vol. 15. Series Comput. Math. Springer, New York (1991).

[8] P.G. Ciarlet, The Finite Element Method for Elliptic Problems. Studies Math. Appl. North Holland, Netherland (1980).

[9] Ph. Clément, Approximation by finite element functions using local regularization. RAIRO Anal. Numér. 9 (1975) 77-84.

[10] P. Coorevits, P. Hild, K. Lhalouani and T. Sassi, Mixed finite element methods for unilateral problems: convergence analysis and numerical studies. Math. Comput. 71 (2001) 1-25.

[11] M. Crouzeix and V. Thomée, The stability in $L^{p}$ and $W^{1, p}$ of the $L^{2}$-projection on finite element function spaces. Math. Comput. 48 (1987) 521-532.

[12] J.St. Doltsinis, J. Luginsland and S. Nölting, Some developments in the numerical simulation of metal forming processes. Eng. Comput. 4 (1987) 266-280.

[13] A. Ern and J.-L. Guermond, Éléments Finis: Théorie, Application, Mise en Oeuvre. Math. Appl. SMAI, Springer 36 (2001).

[14] M. Fortin and D. Côté, On the imposition of friction boundary conditions for the numerical simulation of Bingham fluid flows. Comput. Methods Appl. Mech. Engrg. 88 (1991) 97-109.

[15] H. Fujita, Flow Problems with Unilateral Boundary Conditions. Leçons, Collège de France (1993).

[16] H. Fujita, A Mathematical analysis of motions of viscous incompressible fluid under leak and slip boundary conditions. Math.

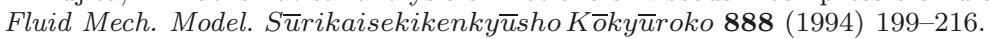

[17] H. Fujita, A coherent analysis of Stokes flows under boundary conditions of friction type. J. Comput. Appl. Math. 149 (2002) $57-69$.

[18] M.K. Gdoura, Problème de Stokes avec des conditions aux limites non-linéaires: analyse numérique et algorithmes de résolution, Thèse en co-tutelle, Université Tunis El Manar et Université de Caen Basse Normandie (2011).

[19] G. Geymonat and F. Krasucki, On the existence of the Airy function in Lipschitz domains. Application to the traces of $H^{2} C$. R. Acad. Sci. Paris, Série I 330 (2000) 355-360,.

[20] V. Girault and P.A. Raviart, Finite Element Approximation of the Navier-Stokes Equations. Springer-Verlag, Berlin (1979).

[21] P. Grisvard, Elliptic Problems in Nonsmooth Domains. Monogr. Studies Math. Pitman (Advanced Publishing Program), Boston, MA 24 (1985).

[22] S.G. Hatzikiriakos and J.M. Dealy, Wall slip of molten high density polyethylene. I. Sliding plate rheometer studies. J. Rheology 3 (1991) 497-523.

[23] J. Haslinger and T. Sassi, Mixed finite element approximation of 3D contact problem with given friction: Error analysis and numerical realisation, ESAIM: M2AN 38 (2004) 563-578.

[24] N. Kikuchi and J.T. Oden, Contact problems in elasticity: a study of variational inequalities and finite element methods. SIAM Studies in Appl. Math. Philadelphia (1988).

[25] Y. Li and K. Li, Penalty finite element method for Stokes problem with nonlinear slip boundary conditions. Appl. Math. Comput. 204 (2008) 216-226.

[26] J.L. Lions and E. Magenes, Non-Homogeneous Boundary Value Problems and Applications. Springer-Verlag, Berlin, New York (1972).

[27] A. Magnin and J.M. Piau, Shear rheometry of fluids with a yield stress. J. Non-Newtonian Fluid Mech. 23 (1987) 91-106.

[28] L. Marini and A. Quarteroni, A relaxation procedure for domain decomposition method using finite elements. Numer. Math. 55 (1989) 575-598.

[29] I.J. Rao and K.R. Rajagopal, The effect of the slip boundary condition on the flow of fluids in a channel. Acta Mechanica $\mathbf{1 3 5}$ (1999) 113-126.

[30] N. Saito and H. Fujita, Regularity of solutions to the Stokes equations under a certain nonlinear boundary condition, The Navier-Stokes Equations. Lect. Notes Pure Appl. Math. 223 (2001) 73-86.

[31] N. Saito, On the stokes equation with the leak and slip boundary conditions of friction type: regularity of solutions. Pub. RIMS. Kyoto University 40 (2004) 345-383,.

[32] E. Santanach Carreras, N. El Kissi and J.-M. Piau, Block copolymer extrusion distortions: Exit delayed transversal primary cracks and longitudinal secondary cracks: Extrudate splitting and continuous peeling. J. Non-Newt. Fluid Mech. 131 (2005) $1-21$. 